\title{
Correction to: The puzzling high velocity G5 supergiant star HD 179821: new insight from Gaia DR2 data
}

\author{
M. Parthasarathy ${ }^{1} \cdot$ G. Jasniewicz ${ }^{2} \cdot F$. Thěvenin ${ }^{3}$
}

Published online: 8 February 2019

(c) Springer Nature B.V. 2019

\section{Correction to: Astrophys Space Sci}

https://doi.org/10.1007/s10509-019-3506-3

Unfortunately the original publication is missing Fig. 1. Please find the figure here:

The online version of the original article can be found under https://doi.org/10.1007/s10509-019-3506-3.

\section{Parthasarathy}

m-partha@hotmail.com

G. Jasniewicz

gerard.jasniewicz@umontpellier.fr

F. Thěvenin

Frederic.thevenin@oca.eu

1 Indian Institute of Astrophysics, Bangalore 560034, India

2 UMR 5299 LUPM CNRS/Universitè Montpellier, Place Eugène Bataillon, CC 72, 34095 Montpellier cedex 05, France

3 Observatoire de la Côte d'Azur, UMR 6202 Cassiopée/CNRS, B.P. 4229, 06304 Nice cedex 4, France

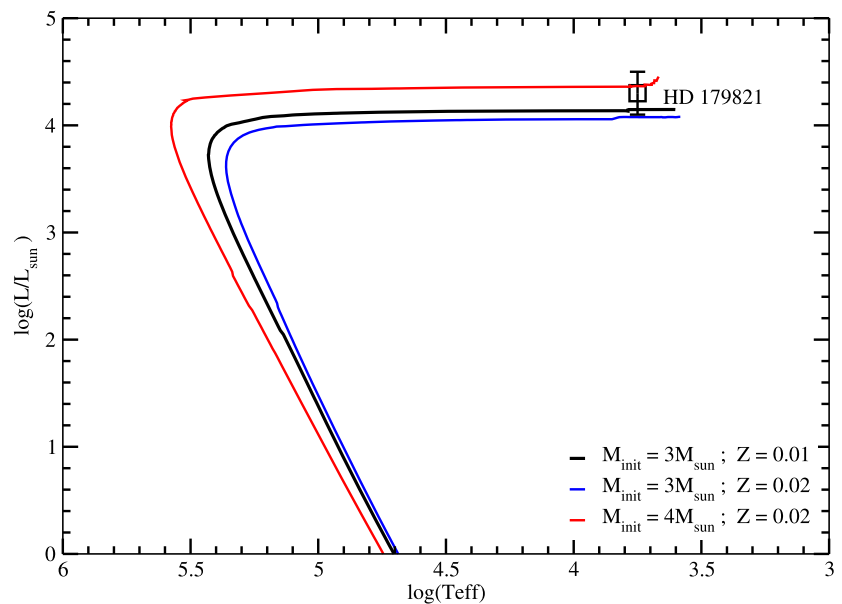

Fig. 1 HR diagram. HD 179821 is located with $T_{\text {eff }}=5660 \mathrm{~K}$ and $\log \left(L / L_{\odot}\right)=4.3 \pm 0.2$ (see Sect. 3 ). The post-AGB models come from Miller Bertolami (2016); the initial masses and metallicities are indicated

Publisher's Note Springer Nature remains neutral with regard to jurisdictional claims in published maps and institutional affiliations.

\section{References}

Miller Bertolami, M.M.: Astron. Astrophys. 588, A25 (2016) 